\title{
As Ações do Serviço de Saúde Voltadas para o Âmbito Individual e Pouco Coletivo
}

\author{
Health Services' Actions Focused on the \\ Individual Rather than Collective Level
}

\author{
Maria Cristina Guimarães da Costa ${ }^{I}$ \\ Chrystinie de Lourdes RamalhãoII \\ Amanda de Gouvêa Pettersen ${ }^{I}$ \\ Julio Augusto Trindade Pio ${ }^{I}$ \\ Sarah Berbare ${ }^{I}$ \\ Vanessa Ferreira Amorim de Melo \\ Yuri Bonicelii Crempe
}

\footnotetext{
PALAVRAS-CHAVE

- Prevenção Primária;

- Atenção Básica;

- Determinação das Necessidades de Saúde;

- Saúde da Família;

- Saúde Coletiva.
}

\author{
KEYWORDS \\ - Primary Prevention; \\ - Primary Health Care; \\ - Health Needs Assessment; \\ - Family Health; \\ - Public Health.
}

Recebido em: 01/04/2010

Aprovado em: 19/11/2010

REVISTA BRASILEIRA DE EDUCAÇÃO MÉDICA 57 36 (1, Supl. 1): $57-63 ; 2012$
${ }^{I}$ Faculdade de Medicina de Marília, Marília, SP, Brasil.

"I Secretaria Municipal de Saúde de Marília, Marília, SP, Brasil.

\section{RESUMO}

Este estudo avalia a compreensão sobre o modelo de atenção básica nas ações de saúde. Trata-se de uma pesquisa de natureza qualitativa exploratória, desenvolvida em Unidades de Saúde da Família (USFs) de Marília, com amostra composta por profissionais de saúde, usuários e estudantes de Medicina e Enfermagem inseridos nas unidades. Os dados foram coletados por meio de entrevistas semiestruturadas e analisados pela técnica de análise de conteúdo, modalidade temática. Pôde-se observar que, independentemente da categoria entrevistada e da área onde se encontra a USF, a compreensão dos entrevistados sobre o modelo de atenção é o modelo médico-assistencial privatista, que reflete o predomínio das ações curativas em uma relação individualista, operada diretamente pelo médico e oferecida na demanda espontânea, não sendo exclusiva do setor privado. Foi proposto utilizar o espaço de grupos organizados para educação em saúde, em que trabalhadores e usuários são participantes para uma interação com as necessidades da comunidade e participação ativa dos usuários no planejamento do serviço, além de investimento nas ações de promoção e prevenção, o que causaria adesão da população e geraria credibilidade no modelo não médico-centrado.

This study evaluates the understanding of the primary healthcare model. This was an exploratory qualitative study conducted in Family Health Units in Marilia, São Paulo, with a sample consisting of health workers, users, and medical and nursing students at the health units. The data were collected with semi-structured interviews and submitted to content analysis (thematic modality). Regardless of the interview category and area where the family health unit was located, the interviewees' understanding of the healthcare model was the private medical care model, which reflects the predominance of curative actions in an individualist relationship, operated directly by the physician and offered according to spontaneous demand, not exclusive to the private sector. The proposal here was to use organized groups as a space for health education in which health workers and users are participants in interaction between the community's needs and active participation by users in planning the service, in addition to investment in health promotion and prevention, intended to foster adherence by the population and generating credibility in a non-doctor-centered model. 


\section{INTRODUÇÃO}

Na última década, várias políticas governamentais foram implementadas com o intuito de promover a inclusão social, com propostas principalmente nas áreas de educação e saúde, sendo que, nesta última, esforços especiais foram realizados para a substituição do modelo técnico-assistencial centrado na doença.

Um dos programas instituídos pelo Ministério da Saúde, por intermédio das Secretarias de Gestão do Trabalho e da Educação na Saúde e Secretaria de Atenção à Saúde, e o Ministério da Educação, por intermédio da Secretaria de Educação Superior, foi o Programa de Educação pelo Trabalho para a Saúde - PET-Saúde, que selecionou Instituições de Educação Superior (IES) para atuar em conjunto com Secretarias Municipais de Saúde.

A Faculdade de Medicina de Marília (Famema), autarquia do governo do estado de São Paulo, é uma instituição voltada para as necessidades da população, que se ocupa em prestar serviços ao município e região e também formar recursos humanos na área de saúde em consonância com os princípios do SUS e as Diretrizes Curriculares Nacionais. A Famema teve oito projetos aprovados para o programa, os quais foram desenvolvidos ao longo do ano de 2009 e encerrados em 2010, cujo propósito foi o de "fomentar grupos de aprendizagem tutorial na Estratégia Saúde da Família, caracterizando-se como instrumento para viabilizar programas de aperfeiçoamento e especialização em serviço, bem como de iniciação ao trabalho, estágios e vivências, dirigidos, respectivamente, aos profissionais e aos estudantes da área da saúde, de acordo com as necessidades do $\operatorname{SUS}^{\prime \prime}$ (p. 606).

Segundo a Constituição Federal de 1988, artigo 196, “a saúde é um direito de todos e um dever do Estado, garantido mediante políticas sociais e econômicas que visem à redução do risco de doenças e de outros agravos e ao acesso universal e igualitário às ações e serviços para promoção, proteção e recuperação"'2(p. 112).

Para fazer valer a Constituição, foi instituído o SUS - Sistema Único de Saúde, que se baseia em princípios éticos de universalidade, integralidade e equidade e nos princípios organizacionais de descentralização, hierarquização e participação social. A sua implementação provocou a necessidade de mudanças nas práticas de atendimento e, com isso, outros programas foram criados, dentre eles o Programa de Agentes Comunitários de Saúde (Pacs), em 1991, considerado o primeiro embrião dessa nova modalidade de prestação de assistência à saúde da população, e o Programa de Saúde da Família (PSF), em 1994, que surge como uma das principais estratégias na consolidação do Sistema Único de Saúde (SUS), por meio da reorganização dos serviços e da reorientação das práticas profissionais.
A estratégia do Programa de Saúde da Família (PSF), como forma de atenção à saúde, teve início na cidade de Marília a partir de 1998, com a implantação de quatro unidades que, após expansão gradativa, têm, hoje, 30 equipes, sendo algumas dessas cenário para residência multiprofissional e inserção de alunos de Medicina e Enfermagem da Famema, que cursam a Unidade de Prática Profissional (UPP). Para o desenvolvimento de tal proposta, a Faculdade conta com a parceria da Secretaria Municipal de Saúde de Marília (SMSM), com a qual compartilha não só a estrutura, mas o processo de trabalho por meio da participação dos profissionais do serviço na construção e desenvolvimento curricular e no acompanhamento dos estudantes. Docentes e estudantes também participam do processo de trabalho das unidades de saúde, de forma a favorecer as discussões e reflexões para a construção de um novo modelo de atenção e de uma nova forma de ensinar.

Os estudantes são estimulados a ter contato com as pessoas, famílias e organizações/equipamentos sociais de uma determinada área do município, com a intenção de compreender as necessidades de saúde na perspectiva da integralidade do cuidado. Essas necessidades não dizem respeito apenas às queixas de sintomas referidos pelo indivíduo, considerando que, nessa perspectiva, a "escuta" será ampliada e as necessidades de saúde se constituirão no centro das intervenções e práticas de saúde ao longo de todo o curso. ${ }^{3}$ (p. 3).

Na Constituição Federal brasileira, que institucionalizou o SUS, inscreveram-se determinantes e condicionantes do processo saúde-doença, reconhecendo, portanto, que saúde não se aprimora somente com a atenção à doença. Com base nessa definição, responder às necessidades de saúde deveria significar implementar ações que incidissem nos determinantes e não só na doença, que já é o resultado do desgaste expresso no corpo biopsíquico individual. Pode-se, então, afirmar que a concepção de saúde-doença expressa no SUS sanciona necessidades de saúde ampliadas. Logo, as respostas deveriam ser mais complexas, para além das ações curativas ${ }^{4}$.

Partindo-se das práticas realizadas ao longo das unidades educacionais e do pressuposto citado anteriormente, passou-se a questionar: quais são as necessidades de saúde que estão sendo apreendidas pelos serviços de saúde? E pelos estudantes inseridos nesse cenário? E pelos usuários que compartilham e utilizam as práticas implementadas?

Assim, a partir desses questionamentos, é que foi proposto o desenvolvimento deste estudo amparado pelo PET-saúde, por meio do qual se pretende buscar a apreensão do conhecimento dos vários atores envolvidos no processo, o que não só possibilitará implementar novas estratégias para a formação acadêmica, como também contribuirá para as reflexões quanto 
à concepção de trabalho sob a óptica de quem "supostamente" produz e de quem consome as ações de saúde.

\section{OBJETIVOS}

Este trabalho é parte de um projeto maior cujo tema era "Necessidades de saúde: um conceito com diferentes entendimentos", fundamental para o cuidado integral na Estratégia Saúde da Família, cujo objetivo era identificar qual o conceito de necessidades de saúde concebido por estudantes, profissionais de saúde e usuários; analisar se o conceito de necessidades de saúde apreendido por esses sujeitos está em sintonia com o ideário do SUS; e contribuir com a reflexão da prática profissional.

\section{MÉTODO}

Trata-se de uma pesquisa de natureza qualitativa exploratória desenvolvida nas Unidades de Saúde da Família (USFs) localizadas nas regiões norte, sul, leste e oeste do município de Marília que tinham inserção de estudantes da $4^{\text {a }}$ série dos cursos de Enfermagem e Medicina da Famema, ,considerando a diversidade socioeconômica das famílias adscritas.

Os sujeitos do estudo foram selecionados de forma aleatória, sendo escolhidas pessoas que fazem parte da equipe das USFs; quatro usuários de cada USF, sendo dois do sexo feminino e dois do masculino, adultos maiores de 20 anos, incluindo idosos; e estudantes da $4^{\text {a }}$ série de Medicina e de Enfermagem que desenvolviam suas práticas nesse cenário. Para garantir o sigilo, as entrevistas receberam um código alfanumérico, sendo A para usuário; B para auxiliar de enfermagem; $C$ para agente comunitário de saúde (ACS); D para médico; E para dentista; F para enfermeiro; G para estudante de Enfermagem; e $\mathrm{H}$ para estudante de Medicina. As entrevistas foram numeradas de acordo com a sequência de inclusão das pessoas em cada categoria, totalizando 36 usuários, nove auxiliares de enfermagem, nove ACSs, oito médicos, nove dentistas, nove enfermeiros, três estudantes de Enfermagem e seis de Medicina.

Após aprovação do Comitê de Ética em Pesquisa Envolvendo Seres Humanos da Faculdade de Medicina de Marília, parecer nº 167/09, e do Conselho Municipal de Avaliação em Pesquisa (Comap) da Secretaria Municipal de Saúde de Marília, foi realizada a coleta de dados, por meio de entrevistas semiestruturadas compostas de duas partes. A primeira englobou dados de identificação e a segunda, questões norteadoras baseadas no objeto deste estudo. As entrevistas foram gravadas após permissão dos sujeitos e transcritas para serem mais bem exploradas.

A análise dos dados foi realizada pela técnica de análise de conteúdo, modalidade temática ${ }^{5}$.

\section{RESULTADOS}

Da exploração do material coletado emergiram sete categorias temáticas: "O processo de trabalho como determinante no processo saúde-doença"; "A construção de espaços participativos para a produção da corresponsabilidade e participação social"; "Necessidades de saúde: aproximações na perspectiva dos sujeitos envolvidos"; "Saúde-doença: contradições de um conceito"; "As interferências das políticas públicas no atendimento nas Unidades de Saúde"; "A reorganização do serviço de saúde e das práticas profissionais como determinantes para ampliar a atenção às necessidades de saúde"; e "As ações do serviço de saúde voltadas para o âmbito individual e pouco coletivo", sendo que este trabalho discorrerá sobre a última temática.

Foi possível observar que, independentemente da categoria entrevistada e da área onde se encontra a Unidade de Saúde, a compreensão sobre o conceito de necessidades de saúde é semelhante e o modelo de atenção é focado no modelo curativo.

A saúde pública/saúde coletiva é definida genericamente como campo de conhecimento e

de práticas organizadas institucionalmente e orientadas à promoção da saúde das populações ${ }^{6}$. Em conformidade com os princípios do Sistema Único de Saúde, o modelo assistencial a partir da atenção básica tende a ser utilizado como estratégia de trabalho da saúde. Nesse modelo, a família e o meio no qual o indivíduo está inserido passam a ser o foco da atenção, e não somente ele é analisado individualmente. Esse modo de intervenção não espera que a população chegue para ser atendida, ao contrário, age preventivamente sobre ela.

No desenvolvimento deste trabalho, pôde-se perceber que nem toda a população compartilha dessas ideias. Usuários e profissionais envolvidos no processo de saúde vigentes nas unidades às quais pertencem diferem de opinião, em sua maioria, em relação ao conceito preconizado pelo SUS. Após realização de entrevista com todas as categorias, percebeu-se que o modelo assistencial integral ainda necessita de maior vinculação e atuação para que atinja o ideário político de saúde.

Nos usuários das Unidades de Saúde entrevistados, observou-se a cultura do modelo assistencialista médico-centrado. Alguns dizem que o que faz falta na unidade são remédios e médicos disponíveis para que haja cobertura nas urgências. Sugerem ainda a possibilidade de atendimento 24 horas, para evitar a sobrecarga hospitalar, e a inclusão de médicos especialistas nas unidades de saúde, alegando a grande demanda. Para a categoria médica, deveriam ser disponibilizados mais médicos na unidade para o atendimento individual, avaliando melhor o contexto em que o paciente vive. De modo geral, voltam o foco da atenção ao cuidado individual, pois este é o modelo que eles trazem em suas referências. 
Dentre os usuários entrevistados, um deles conta que passa por consulta médica a cada quatro meses em instituição particular, onde também realiza exames e adquire medicamentos. Na unidade de saúde, a esposa, portadora de diabetes mellitus, passa por atendimento médico a cada quatro meses e não consegue realizar o controle da doença, pois a Secretaria não retorna os exames realizados por ela. Algumas pessoas citam que vão realizar controle de hipertensão arterial sistêmica, solicitar receitas médicas e adquirir medicamentos. Já uma outra relata que só utiliza o serviço para imunização e prevenção.

Nas respostas das auxiliares de enfermagem e agentes comunitários de saúde (ACS), identificou-se que algumas pessoas procuram a unidade de saúde para fazer prevenção, como exames de rotina. Outras procuram quando estão doentes ou quando estão com dor e a automedicação não apresenta resolubilidade. Os ACSs também afirmam que as unidades necessitam aumentar o número de vagas de encaminhamento para especialidades, além do número de determinados profissionais, assim como o oferecimento de exames complementares. Alguns citam a necessidade do aumento dos locais de pronto-atendimento.

As enfermeiras dizem que a unidade de saúde não atende às necessidades de saúde da população, pois faltam outros profissionais, como psicólogo, assistente social e fisioterapeuta. Dizem também haver falta de insumos, principalmente medicamentos, considerando-se o número de pacientes atendidos. Afirmam que, quando buscam atendimento, o fazem em plantões de pronto-atendimento e que, na maioria deles, focam-se na queixa biológica.

Esse modelo que os entrevistados trouxeram em suas falas, chamado modelo médico-assistencial privatista, predomina no Brasil e está voltado para os indivíduos que, na dependência do seu grau de conhecimento e/ou sofrimento, procuram os serviços de saúde por "livre iniciativa". Consequentemente, aqueles que não percebem seus problemas de saúde ou que não estão alertas para as medidas de prevenção não o fazem. Esse modelo assistencial reforça a atitude dos indivíduos de só procurarem os serviços quando se sentem doentes. Nesses casos, segundo o mesmo autor, os serviços acabam se organizando ou restringindo suas preocupações a manter uma dada oferta de atendimento. “É a pressão espontânea e desordenada da demanda que condiciona a organização de recursos para a oferta. Outras vezes é a própria oferta, distorcida em relação às necessidades de saúde, que determinaria o consumo de serviços médicos"'7 (p. 568).

A partir do momento de criação do Sistema Único de Saúde - SUS, o modelo essencialmente médico-centrado e curati- vista cede lugar a um novo modelo de atuação na saúde, com propostas para mudar toda a antiga concepção de atuação dos profissionais, passando a atuar na integralidade da assistência e olhando o indivíduo em seu âmbito coletivo.

Em 1988, com a promulgação da nova Constituição, estabeleceu-se o lema: "Saúde é direito de todos e dever do Estado", ou seja, todo brasileiro tem garantido por lei o acesso às ações de prevenção, promoção e recuperação da saúde ${ }^{8}$. A Lei 8.080/90, Lei Orgânica da Saúde, promulgada pelo Ministério da Saúde e que regulamenta o SUS, passou a reger várias iniciativas institucionais para desenvolvimento de ações em saúde a partir desse fato. Segundo essa Lei, a saúde não é só a ausência de doenças e é determinada por uma série de fatores presentes no dia a dia, tais como: trabalho, educação, lazer, moradia, saneamento básico, meio ambiente, alimentação, etc. Foi criada também a Lei 8.142/90, que regulamenta a participação da comunidade na gerência do SUS através dos Conselhos e das Conferências de Saúde.

O Programa de Saúde da Família (PSF) teve início quando o Ministério da Saúde formulou, em 1991, o Programa de Agentes Comunitários de Saúde (Pacs) com a finalidade de contribuir para a redução das mortalidades infantil e materna, principalmente nas regiões Norte e Nordeste, por meio da extensão de cobertura dos serviços de saúde para as áreas mais pobres e desvalidas. A partir da experiência acumulada no Ceará com o Pacs, o Ministério da Saúde percebeu a importância dos agentes nos serviços básicos de saúde no município e começou a enfocar a família como unidade de ação programática de saúde, não mais enfocando somente o indivíduo, mas introduzindo a noção de cobertura por família. Assim, o PSF foi concebido a partir de uma reunião ocorrida nos dias 27 e 28 de dezembro de 1993 em Brasília, DF, sobre o tema "Saúde da Família", convocada pelo gabinete do então ministro da Saúde, Henrique Santillo, com apoio do Unicef. A reunião esteve assentada na discussão de uma nova proposta a partir do êxito do Pacs e da necessidade de incorporar novos profissionais para que os agentes não funcionassem de forma isolada ${ }^{9}$.

\section{Demanda nos Serviços de Saúde com Enfoque nas Ações Curativas sobre as Preventivas}

O discurso da saúde pública e as perspectivas de redirecionar as práticas de saúde vêm se articulando em torno do processo de integralidade. Esta se caracteriza pela assimilação das práticas preventivas e das práticas curativas por um mesmo serviço. Assim, o usuário não precisa se dirigir a unidades de saúde distintas para receber atendimento curativo e preventivo. Entretanto, o que tem sido observado nos serviços de saúde, e que também foi verificado com as entrevistas realizadas, 
é que as ações desenvolvidas de acordo com as demandas nos serviços estão focadas predominantemente no modelo curativo. A prevenção é mencionada por alguns dos entrevistados tanto como ação não desenvolvida nas unidades quanto como alternativa de melhora nos atendimentos de saúde, apontando para uma população consciente da importância do trabalho preventivo, mas que ainda modela suas ações sob o caráter curativo.

É possível inferir que a estratégia do PSF buscou modificar a organização das unidades de saúde, porém não necessariamente transformou as suas práticas de forma a articular a prevenção e a assistência conforme proposto pelo SUS.

A demanda pela assistência, que ocorre a partir de uma experiência de sofrimento de uma pessoa, e a "demanda espontânea", em que o indivíduo compreende ser possível encontrar alento para tal sofrimento em um serviço de saúde, são construídas socialmente, embora experimentadas individualmente. Já as ações preventivas "se antecipam à experiência individual de sofrimento. Elas atuam ainda no silêncio dos órgãos" e são possíveis porque, com os conhecimentos dos profissionais de saúde, é possível antever riscos ou mesmo reconhecer a doença antes mesmo que ela gere sofrimento. Portanto, a necessidade de uma ação preventiva é ditada a partir do conhecimento dos profissionais e, de certa maneira, ofertada por eles. Entende-se aqui que articular a demanda espontânea aos procedimentos preventivos é uma das dimensões da integralidade ${ }^{10}$.

Para os profissionais, isso significa incluir "no seu cotidiano de trabalho rotinas ou processos de busca sistemática daquelas necessidades mais silenciosas", criar estratégias no serviço e adotar processos de trabalho que permitam oferecer ações voltadas para a prevenção. Isso não significa puramente criar protocolos ou rotinas para identificar necessidades não demandadas pela população. Há que se adotar uma postura que identifique, a partir do conhecimento técnico, as necessidades assistenciais e de prevenção e que selecione as intervenções a serem ofertadas no contexto de cada encontro entre os membros da equipe e as pessoas que procuram o serviço ${ }^{10}$.

Diversos fatores de melhora do atendimento nas unidades de saúde são apontados pelos entrevistados, e a ação preventiva é citada por muitos, mostrando a conscientização deles quanto à percepção da prevenção como alternativa de melhora no serviço. A categoria das enfermeiras cita a prevenção, dentre outros fatores, como fator de melhora no atendimento. Para elas e para os estudantes de Enfermagem, há a crença de que a melhora do planejamento local nas USFs, a prevenção e educação em saúde, o olhar do indivíduo como um todo, a capacitação dos profissionais e a maior oferta de vagas para as especialidades seriam essenciais para a melhora do atendimento.

Os dentistas apontaram como sugestões de melhora a diminuição do fluxo de atendimento para terem mais tempo de trabalhar a prevenção e a promoção da saúde e de realizarem um atendimento multiprofissional com maior qualidade, em que o paciente possa ser visto como um todo. Com isso, o acolhimento seria mais completo, no intuito de buscar a integralidade das ações. Eles também informaram em suas entrevistas que, atualmente, conseguem trabalhar mais no modelo curativo do que no preventivo e de promoção à saúde.

No que se refere às políticas de saúde, caberia apontar processos de mudança nos procedimentos técnicos de prestação de serviços, no uso das tecnologias disponíveis, na integração de saberes e conhecimentos, bem como nas formas de utilização dos recursos e de concretização da participação social. Nessa perspectiva, as diretrizes do SUS estão exigindo um enfoque mais global das ações de saúde agrupadas de acordo com o conjunto dos problemas da população, sendo executadas de modo integral, evitando-se as intervenções por patologias ou programas isolados. Os serviços deveriam ser organizados para produzir mudanças no perfil epidemiológico, a partir das necessidades e problemas de saúde (riscos e danos) coletivos e individuais, valendo-se de modelos assistenciais mais abrangentes que articulam ações preventivas, curativas e promocionais de saúde ${ }^{7}$.

As necessidades de saúde podem ser expressas por demandas, como uma consulta médica, o consumo de medicamentos e a realização de exames; entretanto, podem ser também a busca de respostas para as más condições de vida que a pessoa viveu ou está vivendo; a procura de vínculo afetivo, ou outras que de alguma forma precisam ser traduzidas e escutadas pela equipe de saúde, configurando, assim, a integralidade da atenção no espaço de cada unidade de saúde ${ }^{11}$.

As ações preventivas definem-se como meios para a diminuição do surgimento de determinadas situações no cenário da saúde ou, pelo menos, para evitar que o indivíduo esteja despreparado para lidar com problemas reais caso tenha que vivenciá-los. As ações próprias dos sistemas de saúde precisam estar articuladas à equipe multiprofissional e a políticas governamentais responsáveis por outros setores que irão influenciar na saúde. O conceito de necessidades de saúde, que para ser contemplado necessita dessa intersetorialidade, utiliza-se da prevenção como um de seus principais meios. Os projetos de prevenção em saúde moldam-se diante da divulgação de informação científica e de recomendações normativas de mudanças de hábitos, sempre no intuito de buscar que as necessidades de saúde da população sejam atendidas. 
Para atender às necessidades de saúde, na opinião das enfermeiras, seria necessário adequar o quadro de funcionários e a sua qualificação, melhorar a qualidade de atendimento da população, implementar a educação permanente, favorecer os direitos da população de acesso a serviços especializados, vagas para atendimento e insumos, e voltar à proposta de equipe multiprofissional.

Abarcando a opinião das auxiliares de enfermagem entrevistadas, a unidade atende às necessidades mais no que se refere à parte curativa e, mesmo assim, parcialmente, pois se pudesse ter mais profissionais, o paciente se deslocaria menos para um serviço referenciado. Ainda, para elas, a população não está acostumada com a proposta do PSF, justificando que, por ser a unidade de saúde anteriormente uma UBS, não há familiaridade com o trabalho de prevenção.

Para atender às necessidades de saúde, na opinião das auxiliares de enfermagem, seria necessário fazer a atenção primária com prevenção à doença e promoção à saúde e também ter mais pronto-atendimentos na cidade, para que a Unidade de Saúde da Família deixasse de atender urgências.

As necessidades de saúde, embora possam expressar outras demandas que não as queixas do doente, tendem a ser vistas como a expressão dos problemas do corpo, sendo esse o principal motivo que leva à organização do serviço de saúde ${ }^{11}$.

Quando se lida com a organização dos serviços de saúde, as necessidades de saúde são aspectos importantes, pois embasam a configuração geral de sua estrutura. Quando se pensa em necessidades de saúde, logo se lembra de "assistência", porque a imagem mais clara está representada pela procura de cuidados médicos, o que faz uma pessoa buscar o serviço quando está doente. A origem dessa busca é algo que o indivíduo entende que precisa ser corrigido em seu estado "sociovital" e é considerada a demanda. Entretanto, também é possível se antever uma necessidade com base no que já se viu em outras pessoas na sociedade ${ }^{12}$.

Quando questionados sobre as situações em que procuram o serviço de saúde, alguns entrevistados citam a prevenção, mas a maioria só busca o atendimento em situações de demanda curativa.

Além da prevenção, os enfermeiros entrevistados buscam o serviço de saúde quando não dão conta das necessidades biológicas mais graves, no âmbito curativo.

Os médicos alegam que só procuram por atendimento na unidade em situações agudas, quando estão muito doentes. A prevenção surge de forma mais discreta, sendo citados o acompanhamento pré-natal e a prevenção de complicações. Esta mesma categoria coloca que a unidade de saúde só consegue suprir o atendimento da doença, que fica mais na parte curativa, principalmente devido ao excesso de demanda.

Os estudantes de Medicina e Enfermagem costumam procurar o serviço de saúde quando se sentem doentes fisicamente, quando estão psicologicamente em situação de limitação ou urgência, ou em qualquer outra situação em que sintam a necessidade de procurar pelo atendimento. Os dentistas apontam que buscam o atendimento em situações de prevenção ou dúvidas referentes à sua doença e também em situações de urgência, mesmo sabendo e orientando as pessoas quanto à importância da prevenção.

Portanto, a reflexão é sobre o predomínio das ações curativas em uma relação individualista operada diretamente pelo médico e oferecida na demanda espontânea, o que, como se pode perceber, não é exclusivo do setor privado.

\section{CONSIDERAÇÕES}

Este estudo permitiu concluir que os profissionais atuantes na rede básica de saúde encontram-se diante de desafios que dificultam a consolidação do novo modelo técnico-assistencial em saúde. A excessiva demanda espontânea e o insuficiente número de profissionais apontados pelos entrevistados, aliados a uma prática curativista hegemônica, configuram-se como um desafio para a realização de ações de prevenção e de promoção da saúde. A reflexão dos entrevistados indica que a oferta de serviços é estruturada de acordo com as demandas destinadas à cura e, em menor quantidade, à prevenção de agravos, com pouca articulação entre si.

Assim, faz-se necessária uma reflexão: a busca de modelos de intervenção mais abrangentes requer, de um lado, uma reflexão sobre os fundamentos teóricos e epistemológicos que sustentam as novas propostas de ação e, de outro, uma análise escrupulosa da situação concreta, no sentido de contextualizar cada intervenção concebida para que possa produzir os efeitos desejados sobre a realidade ${ }^{7}$. Cabe inferir que uma proposta de intervenção seria ações voltadas à educação em saúde, abrangendo uma maior capacitação dos profissionais, estudantes da área de saúde e também usuários das unidades de saúde, para que eles pudessem estar mais familiarizados com o modelo vigente, pois muitos se mostraram afastados dos conceitos atuais. Não é possível desenvolver um determinado modelo assistencial se os envolvidos no processo não estão a par dos fundamentos. Além disso, a estratégia do PSF reconhece a necessidade de reorientação das práticas de saúde e a renovação dos vínculos de compromisso entre os serviços e a população assistida. O desenvolvimento de um novo modelo assistencial com base nos princípios do PSF não implica um retrocesso quanto à incorporação de tecnologias avança- 
das. Tal proposta demanda a reorganização dos conteúdos dos saberes e práticas de saúde, de forma que eles reflitam os pressupostos do SUS no cotidiano dos profissionais e no atendimento das necessidades de saúde da população.

No sentido de buscar tentar solucionar os problemas levantados nesta pesquisa, encarou-se como alternativa a proposta de dar voz aos usuários na relação com os trabalhadores de saúde, tomando a participação social como um processo construído coletivamente, por meio da estratégia de mostrar como determinadas formas de conversação podem potencializar o vínculo e a corresponsabilização na saúde.

Assim, a intervenção proposta é utilizar o espaço de grupos organizados para educação em saúde, em que trabalhadores e usuários são participantes, buscando uma forma mais dialógica de funcionamento que favoreça uma interação com as necessidades da comunidade e uma participação mais ativa dos usuários no planejamento do serviço de saúde.

Soma-se às alternativas apresentadas um investimento maior na efetividade das ações de promoção e prevenção de saúde, o que causaria uma maior adesão da população e geraria maior credibilidade ao modelo não médico-centrado.

\section{REFERÊNCIAS}

1. Brasil. Ministério da Saúde. Secretaria de Gestão do Trabalho e da Educação na Saúde Seleção para o Programa de Educação pelo Trabalho para a Saúde - PET-Saúde Edital no 12 , de 3 de setembro de 2008. Diário Oficial da União. Brasília, 04 set. 2008; Seção 3.

2. Brasil. Constituição da República Federativa do Brasil: promulgada em 5 de outubro de 1988. 4. ed. São Paulo: Saraiva; 1990 (Série Legislação Brasileira).

3. Faculdade de Medicina de Marília. Necessidades de saúde 1: $1^{\underline{a}}$ série - Cursos de Medicina e Enfermagem. Marília: Famema; 2008.

4. Campos CMS, Bataiero MO. Necessidades de saúde: uma análise da produção científica brasileira de 1990 a 2004. Interface Comunic Saúde Educ. 2007;11(23):605-18.

5. Gomes R. A análise de dados em pesquisa qualitativa. In: Minayo MCS, org.), Pesquisa social: teoria, método e criatividade. ; 25a ed. Petrópolis: Vozes; 2007. p. 79-108.

6. Sabroza P C. Saúde pública: procurando os limites da crise. Rio de Janeiro: Fiocruz;1994.
7. Paim J S. Modelos de Atenção e Vigilância da Saúde. In: Rouquayrol, M Z , Almeida.Filho N. Epidemiologia \& Saúde. , 6 ${ }^{\mathrm{a}}$ ed. Rio de Janeiro; MEDSI.; 2003. p. 567-86.

8. Brasil. Ministério da Saúde. Assessoria de Comunicação Social, ABC do SUS/MS. 2a ed. Brasília (DF): MS; 1991.

9. Rosa WAG, Labate RC. Programa Saúde da Família: a construção de um novo modelo de assistência. Rev Latino-Am Enfermagem. 2005;13(6):1027-34.

10. Mattos R A. A integralidade na prática (ou sobre a prática da integralidade). Cad Saúde Pública [periódico na internet]. 2004 [acesso em 15 mar. 2010];20(5):1411-6. Disponível em: http://www.scielo.br/scielo.php?script=sci_artte xt\&pid=S0102311X2004000500037\&lng=en\&nrm=iso.

11. Cecílio LCO. As necessidades de saúde como conceito estruturante na luta pela integralidade e equidade na atenção em saúde. In: Pinheiro R, Mattos, R A, org. Os sentidos da integralidade na atenção e no cuidado à saúde. ; $4^{\mathrm{a}}$ ed. Rio de Janeiro: ABRASCO; 2006. p.113-126.

12. Schraiber L B, Mendes-Gonçalves RB. Necessidades de saúde e atenção primária. In: Schraiber L B, Nemes,M IB, Mendes-Gonçalves RB, org. Saúde do adulto: programas e ações na unidade básica. São Paulo: Hucitec; 1996. p. 29 $-47$.

\section{CONTRIBUIÇÃO DOS AUTORES}

Todos os autores contribuíram na concepção do manuscrito, obtenção, análise e interpretação dos dados; contribuíram significativamente na elaboração e revisão crítica do mesmo e participaram na revisão da versão final.

\section{CONFLITO DE INTERESSES}

Declarou não haver.

\section{ENDEREÇO PARA CORRESPONDÊNCIA}

Maria Cristina Guimarães da Costa

Av. Itu, 102 ap. 701

Bairro Cascata - Marília

CEP: 17515-030 SP

E-mail: mcgcosta@superig.com.br 